\title{
Real Time Monitoring Of Urban Water Systems for Developing Countries
}

\author{
Offiong N. M. ${ }^{1}$, Abdullahi S. A. ${ }^{1}$, Chile-Agada, B. U. N. ${ }^{2}$, Raji-Lawal H. Y ${ }^{3}$, \\ Nweze N. O. ${ }^{1}$ \\ Correspondence:*nsikakm@nsuk.edu.ng \\ 1. Department of Mathematical Sciences, Nasarawa State University, Keffi, Nasarawa, Nigeria. \\ 2. Department of Computer Science, Alvan Ikoku Federal College of Education, Owerri, Imo, Nigeria. \\ 3. Department of Computer Science, Lagos State University, Ojo, Lagos, Nigeria.
}

\begin{abstract}
The safe and satisfactory provision of water related services in urban areas of developing countries remain a major challenge mainly attributed to limited resources and inability to promptly detect operational failures (blockage, leakage, organic infiltration etc..) in water infrastructure. In this paper we investigated the feasibility of real time monitoring for contexts similar to developing countries and developed a decision support tool to prioritizeloptimize water management practice. Wireless sensors were use to generate data and identification of opportunities /challenges/strategies for setting up a viable real time monitoring mechanism. The application of wireless sensor networks is composed of a number of sensor nodes that have the capability of carrying out network activities and can work continuously for the sake of monitoring. This paper proposes how urban water monitoring systems for developing countries and emerging economies can be developed with emphasis on cost effectiveness, easy ad hoc installation such that the mechanism will be easy to handle and maintained. The use of wireless network for this purpose reduced the manpower required, set up cost and provided flexibility in terms of distance. The paper made use of Zigbee technology because of its low power consumption and it can be used to realize water quality parameters, remote probing and real time monitoring function. Several results are presented and the feasibility of using wireless sensor network is presented.

Keywords: Real-Time, Monitoring, Water Systems, Developing Countries.
\end{abstract}

\section{Introduction}

With consistent outbreak of water borne diseases in developing countries, it becomes imperative to come up with effective water monitoring systems for water infrastructure and water bodies of developing nations. These water monitoring systems should be capable of monitoring water bodies, taking note of possible contaminants based on certain data supplied and give feedbacks at real-time. At present, water pollution is ravaging the sub-Saharan region and the developing parts of Asia [4]. The pollution in turn comes with waterborne diseases which are detrimental to the lives of the people in these regions.

Most of the small water catchment areas in Africa and especially Nigeria need continuous monitoring for microbial contamination. It is most severe for those water resources used for irrigation and swimming purpose. Currently, this is done by manually sampling the water bodie; requiring a person to collect the sample and take it to the lab for testing. Daily, developing nations are making progress economically and industrially and this progress has resulted in pollution of the natural water habitat and water bodies, thereby posing a challenge to the existence of flora and fauna. Authorities have set up various environmental monitoring agencies, but limited resources, terrorist activities and operational policies are affecting the effective monitoring of the water bodies and habitats. In recent years, water monitoring systems have attracted a lot of attention globally, [1] and researchers are looking at various methods of monitoring water bodies with the aim of coming up with a viable, cost-effective water monitoring infrastructure.

At the moment in Nigeria, monitoring water bodies is hazardous owing to poor access to the creeks and seeming dangers of pirates. In the light of this, this paper takes a look into wireless technology to bring out ways they can be harnessed in water quality monitoring. It describes how Zigbee technology, wireless sensor networks and GPRS can be used to build a parallel distributed network using mesh topology for urban water quality monitoring in developing countries and emerging economies. With developing countries and emerging economies in mind, there is a need for systems that allow for timely warning and information on water pollution and pathogens and on the total state of the water system. Furthermore, suitable instruments for accurate process control and in the field environmental monitoring need to be developed. For source water and environmental monitoring as well as process control instruments are needed that can be employed on location or in-situ and that can be monitored through remote control. Low maintenance, affordability and performance (accuracy, precision and speed) are further highly important requirements that new sensing technologies need to meet. 
Real time monitoring tools and models are required within the whole water cycle; be it water resources management, water for people, water in industry and water in agriculture. The quality of the water should be tailored to the specific needs for human, industrial and agricultural consumption. Systems are needed for timely warning and information on pollution and pathogens, be it accidental or in the course of terrorist attacks. This includes online and 'at the site' monitoring and early warning systems but also test kits for toxicity, pathogens and contaminations. Recent advances in molecular biology and nanotechnology has made it possible to develop a set of monitoring systems which deliver reliable information at a much faster pace than the classical methods, and which can be applied even for remote control.[5]

\section{WATER QUALITY}

The term water quality is used to describe the condition of the water, including its chemical, physical and biological characteristics, usually with respect to its suitability for a particular purpose (i.e., drinking, swimming or fishing). Water quality is also affected by substances like pesticides or fertilizers that can negatively affect marine life when present in certain concentrations. The following factors are often used to provide a measure of water quality: concentration of dissolved oxygen (DO); levels of fecal coliform bacteria from human and animal wastes; concentrations of plant nutrients nitrogen and phosphorus; amount of particulate matter suspended in the water (turbidity); and amount of salt (salinity). In many bodies of water, the concentration of chlorophyll-a, a green pigment found in microscopic algae, is also filtered from water samples to give a measure of the microalgae living in the water column. Quantities of pesticides, herbicides, heavy metals and other contaminants may also be measured to determine water quality. [3]

\section{Monitoring Systems}

R. Madhan et al. [8] developed a new patented water monitoring device known as AVP. The device was demonstrated on fresh water body using high-resolution time series profiling by the patented AVP. The shortfall is that the device lasted for a maximum period of nine (9) days transmitting data over a 50KM distance. Marco Zennaro et al. proposed a water quality wireless sensor network (WQWSN) which was applied to water quality in Malawi. The WQWSN is built on the sunSPOT technology, a prototype implementation of water quality wireless sensor network (WQWSN) as a solution to water quality monitoring problem. They used the three layer architecture to discuss the features of WQWSN. Here, their aim is to mitigate two challenges which include: energy consumption of the system and internetworking of the system. Another point of concern here is that the model did not capture some other parameters as will be discussed.

Currently, the feasibility of molecular-based monitoring methods is being tested. This study investigated the feasibility of using a reusable, flow-through nucleic acid extraction and quantitative PCR (pPCR) module to detect human pathogens using previously published qPCR assays for Enterococcus and human bacteriodes. The module when integrated into the Environmental Sample Processor creates an in situ platform that allows autonomous sampling and analysis of seawater for potential human pathogens. The qPCR assays were tested against a library of $16 \mathrm{~S}$ rRNA genes from bacteria commonly found in surface waters of Monterey Bay for testing their use in non-point source contamination [7].

\section{ZIGBEE TECHNOLOGY}

ZigBee is a technology is a small range, low power and low data rate wireless networking technology for wireless applications. It can be found at the bottom three layers of the seven layers of a network model and these includes the physical, data link and network layer. This is the recently published IEEE 802.15.4 standards for personal area networks. ZigBee is embedded with radio-frequency (RF) applications that require a low data rate, extended battery life, and secure networking. ZigBee is a wireless technology developed as an open global standard to address the unique needs of low-cost, low-power, wireless sensor networks.

Zigbee devices are physical objects that are equipped with a radio for transmission and communication purpose. It is composed of a set of intercommunication devices which aid in the implementation of an application such as automation systems.

The physical, MAC and network layers are used to create and maintain the communication network that are interconnecting each Zigbee devices. The application support layer is used to communicate information between devices. [6]

The IEEE 802.15.4 (ZigBee) standard provides three frequency bands for operation: these are $868 \mathrm{MHz}$, $916 \mathrm{MHz}$, and $2.4 \mathrm{GHz}$ for ZigBee. $868 \mathrm{MHz}$ band used in only Europe and has the 20Kbps data rate of transmission and contain only one channel with BPSK modulation technique. $916 \mathrm{MHz}$ band is used in Americas having the $40 \mathrm{Kbps}$ data rate of transmission and contain 10 channels with BPSK modulation technique. $2.4 \mathrm{GHz}$ frequency bands used throughout the world because of ISM (Industrial, Scientific, Medical) band. It has $250 \mathrm{Kbps}$ data rate of transmission and 16 channels with O-QPSK modulation technique. Transmission distance 
is within the range from 30 meters in an indoor non-line of sight of environment and 100 meters in line of sight environment. The range problem can be solved by using various routing algorithms at the network layer.

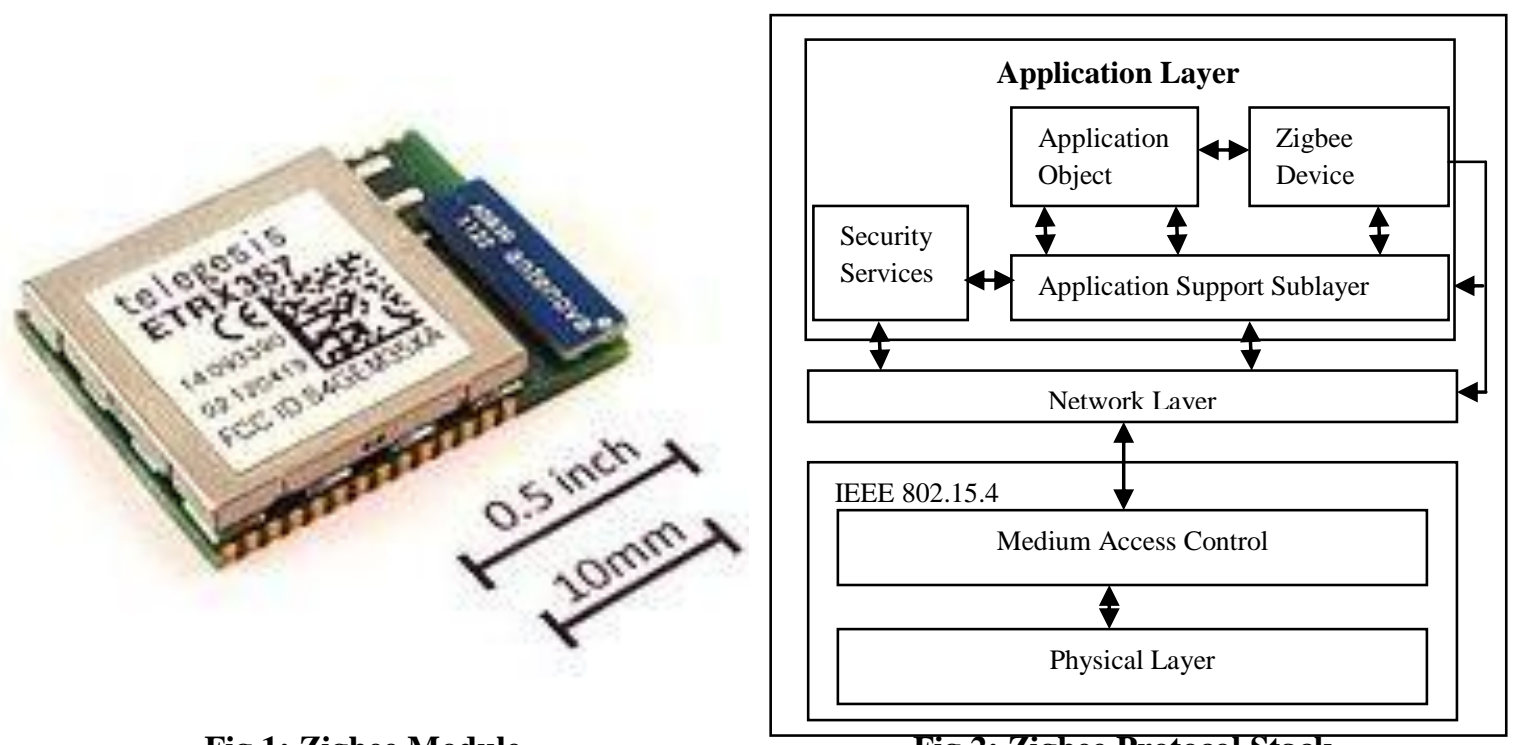

Fig 1: Zigbee Module

Fig 2: Zigbee Protocol Stack

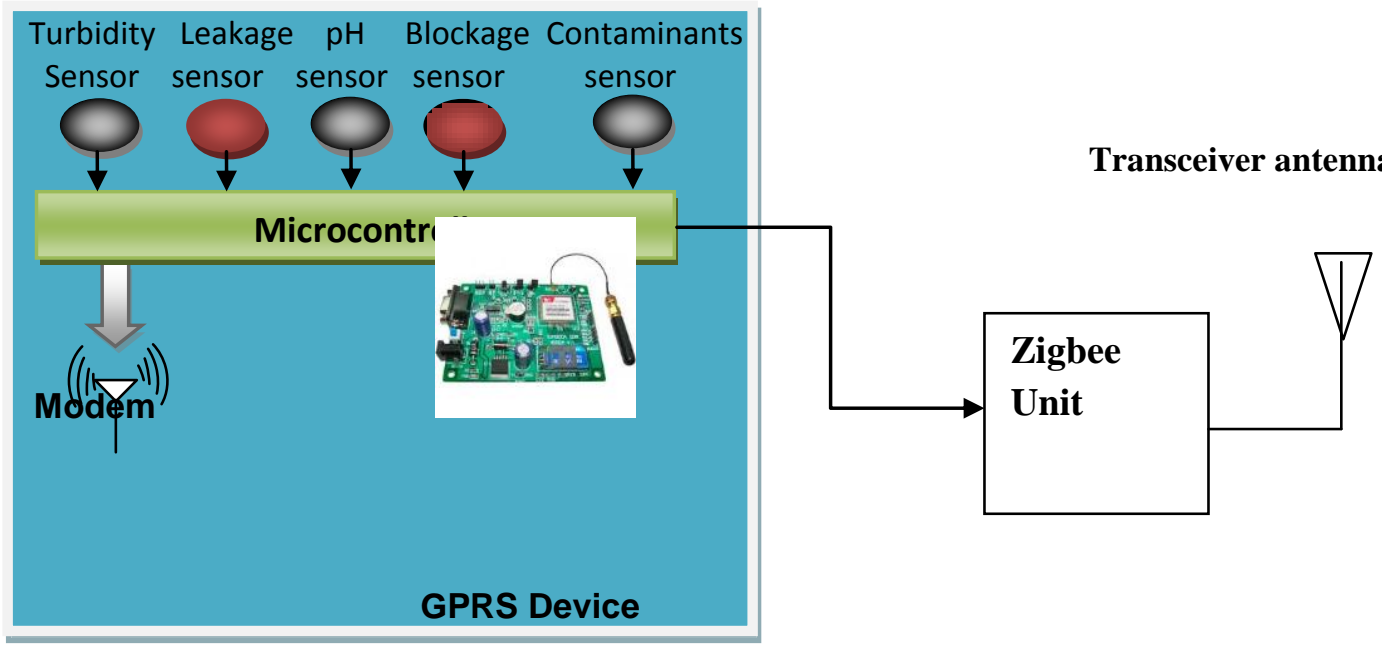

Fig 3: Hardware Design of UWQMS

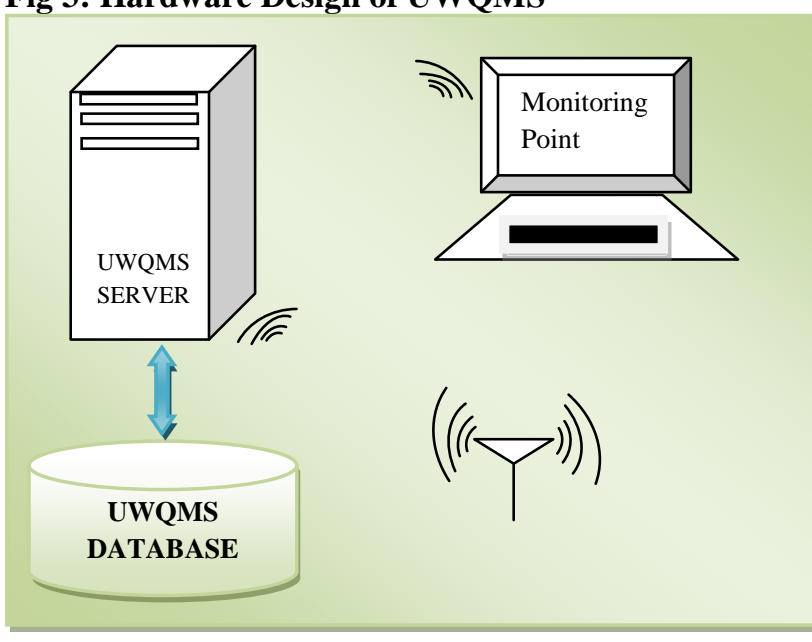

Fig 4: UWQMS Monitoring Station UWQMS DESIGN

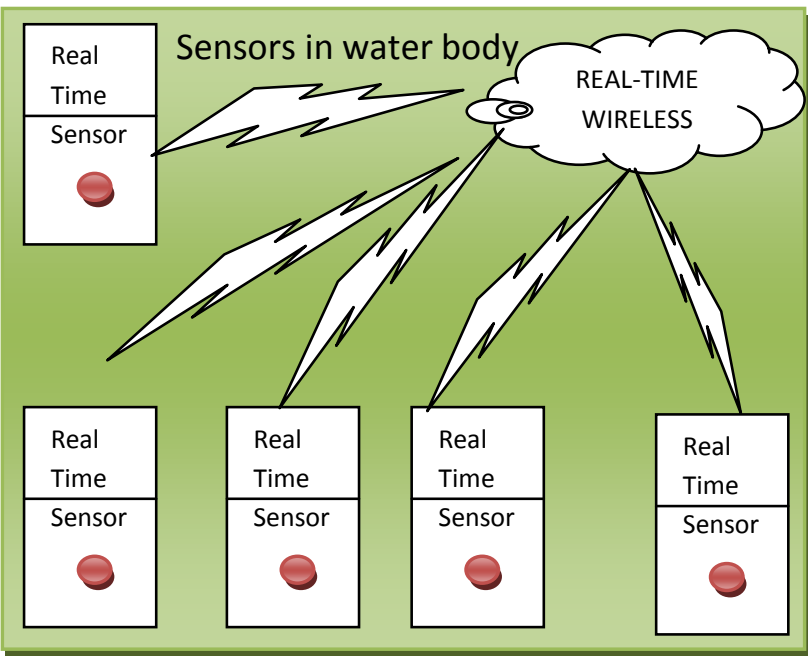

Fig 5: Distribution of Sensors at water body 
For the purpose of this work, we designed a monitoring system that is peculiar to developing countries putting into consideration factors such as: power supply, cost of setting up the infrastructure etc. In the design, we used Zigbee technology to a reasonable extent and the reasons are as follows:

- Zigbee maximizes effective communication range and does not require a specialized computer or central access point and increases robustness and reliability of information

- The network design topology adopted in the design is mesh topology because of the fact that each node on the network is a redundant access point. This is basically because a central node cannot be relied upon. Zigbee becomes more efficient here.

- Zigbee has a defined rate of $250 \mathrm{kbits} / \mathrm{s}$, which is best suited for periodic data or a single signal transmission from a sensor or input device.

- Zigbee network are secured by 128 bit symmetric encryption keys.

At the water body, we deployed a series of sensors to send data on the $\mathrm{pH}$ level of the water body, contaminants and turbidity. In the water transmission pipes, we inserted sensors to monitor pressure and leakages where necessary. The sensors were randomly distributed with no central node. When a parameter is sensed, it is transmitted to the UWQMS station and the data acted upon and stored in the database for future use or record purpose. Microelectromechanical systems (MEMS) were attached to the sensor because it combines the technology of computers with tiny mechanical devices. Memosens is a technology that can be used with any type of analytical instrument. Memosens make the sensors intelligent and is capable of storing and transmitting data in digital form to the receiving point.

The real-time continuous sensor for detection of contaminants in the water body consists of one sample management module, including a mechanism to control sample flow management to the monitoring point. One electronic module for data processing and control is provided, and there is at least one optical module consisting of at least one real-time replaceable sensor cartridge containing a plurality of sensors, and at least one real-time optical pathogen detector connected to the electronic module for data processing. Power is provided and the device is equipped with one secure communication module adapted to transmit encrypted information over a secure link to monitor location and for receiving information. The UWQMS also discloses a system making use of detectors for real-time detection of contaminants and for early warning capability.

\section{Sensors used on UWQMS}

\begin{tabular}{|l|l|l|}
\hline Sensor & Accuracy & Response Time \\
\hline Turbidity & $0-25 \mathrm{NTU}$ & $<125 \mathrm{~ms}$ \\
\hline $\begin{array}{l}\text { Contaminants } \\
\text { (Dissolved Oxygen) }\end{array}$ & $0-200 \% \mu \mathrm{M}$ & $1 \mathrm{~s}$ \\
\hline $\mathrm{pH}$ & 7.4 & $<1 \mathrm{~s}$ \\
\hline
\end{tabular}

\section{Conclusion}

The purpose of this paper is to show a water monitoring system that is peculiar to developing nations and emerging economies, considering cost of infrastructure, maintenance, poor power supply and lack of trained personnel. The work made effective use Zigbee technology and some specialized sensors for detecting several parameters and sending such data to the monitoring point for further action as may apply. This paper gives clear ideas on how to monitor water in real time measuring in an urban area to ultimately improve the water quality of urban dwellers.

\section{Reference}

[1]. Chris Scholin, (2009). "Real-time water quality monitoring" http://www.mbari.org/education/internship/09interns/papers/Frazier.pdf. Retrieved 23 April, 2014.

[2]. Darshana N. Tambe and Ms. Nikita Chavan (2013) "Detection of Air Pollutant Using Zigbee" International Journal of Ad hoc, Sensor \& Ubiquitous Computing (IJASUC) Vol.4, No.4, August 2013

[3]. Diersing, Nancy (2009). "Water Quality: Frequently Asked Questions." Florida Brooks National Marine Sanctuary, Key West Florida.

[4]. JMP, Water for Life: making it happen,2005http://www.wssinfo.org/pdf/JMP_05_text.pdf

[5]. Totti Konnola (2006). "Future Outlook on Water Quality Monitoring Technologies" VTT Technical Research Centre of Finland

[6]. "Understanding the 802.15.4 and Zigbee Network". http://www.daintree.net/learning. Retrieved 13:24hrs, April 22, 2014.

[7]. ZHAO Lian-qing, LI Hai-tao et al. (2008), "Application of Ethernet in Embedded-system Electric Power Remote Terminals for Automatic Meter Reading," China Rural Water and Hydropower , 2008, pp. 78-80.

[8]. Madhan R. et al (2012) "Profiling Device to Monitor Remote Water Bodies" Current Science, vol. 102, no. 2, 25 January 2012 